\section{Fluoroscopically guided removal of a retained sheared guidewire following failed endoscopic ultrasound-guided pancreatic duct drainage}



Fig. 1 Computed tomographic scan showing a retained sheared 0.035 -in guidewire in the pancreatic duct and parenchyma of a 50 -year-old man after a previous failed attempt at endoscopic ultrasound-guided pancreaticogastrostomy.
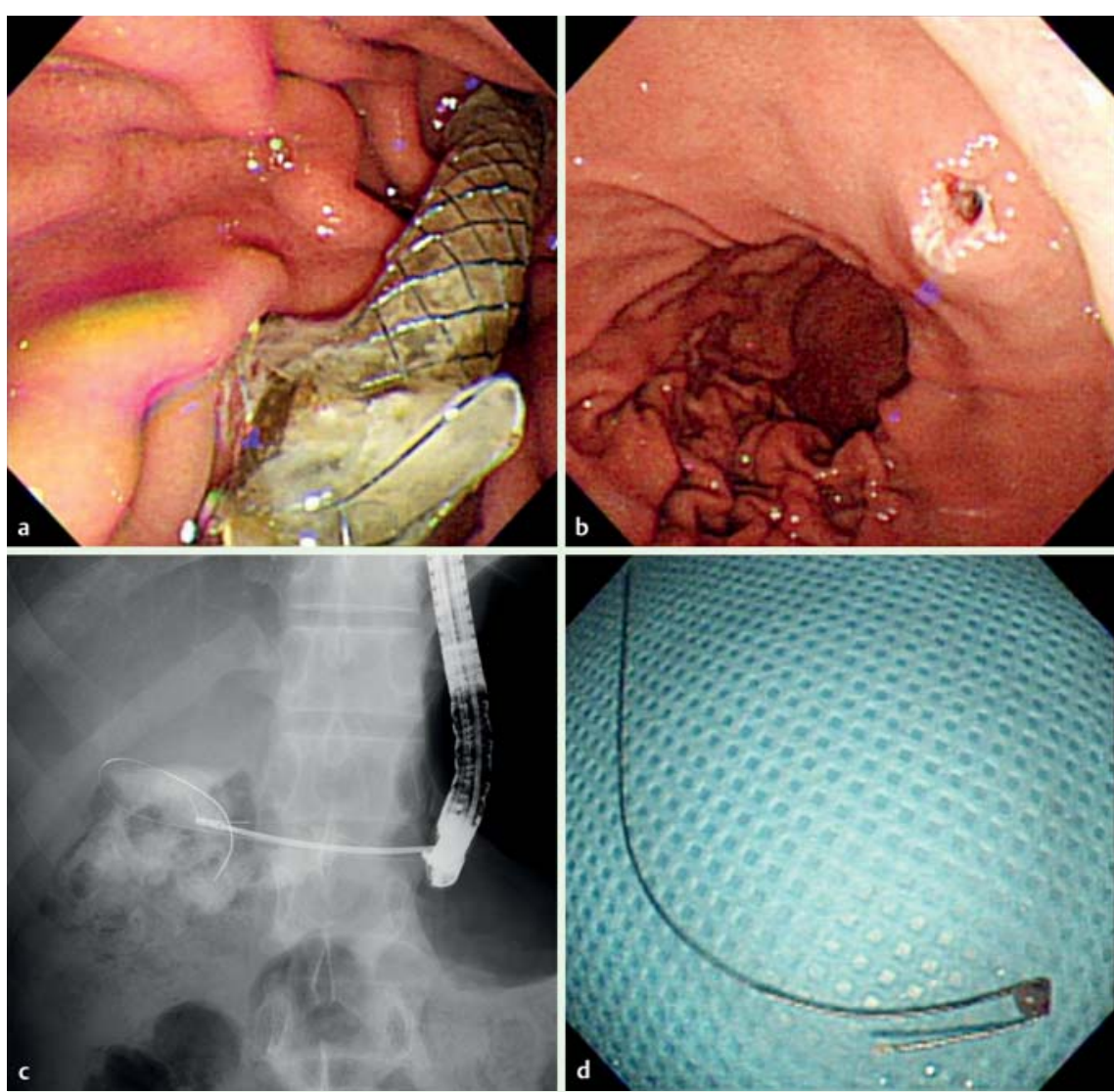

Fig. 2 a Endoscopic image of fully covered, self-expanding metal stent placed by pancreaticogastrostomy. $\mathbf{b}$ Endoscopic image of the high profile fistula opening after removal of the metal stent. c Fluoroscopic image showing successful grasping of the retained guidewire with a rat-tooth forceps. d The removed 7-cm sheared guidewire.
A 50-year-old man with a retained sheared guidewire in the main pancreatic duct and pancreatic parenchyma was referred for recurrent pancreatitis. He had previously undergone a pancreaticoduodenectomy for pancreatic hemangioma and was experiencing recurrent pancreatitis due to pancreaticojejunostomy stricture. Endoscopic ultrasound-guided pancreaticogastrostomy (EUS-PG) had been attempted at another hospital. However, this procedure had failed, and part of a 0.035-in guidewire (Roadrunner; Cook Medical, Bloomington, Indiana, USA) was retained in the pancreatic duct and pancreatic parenchyma ( $\bullet$ Fig. 1 ).

At our hospital, EUS-PG revealed a stricture in the pancreaticojejunostomy through which the guidewire could not pass. A fully covered, self-expandable metal stent, $6 \mathrm{~mm}$ in diameter and $8 \mathrm{~cm}$ in length (M.I.Tech, Seoul, Korea) was placed at EUS-PG.

The patient was readmitted for removal of the retained guidewire and stent revision 6 weeks after the transmural stenting. After removal of the metal stent, pancreatography conducted through the fistula showed a decompressed pancreatic duct. Because the tract had matured as a result of use of the metal stent, a rat-tooth grasping forceps (FG-8L-1; Olympus, Tokyo, Japan) could move freely through the pancreatic duct under fluoroscopic guidance with a 0.025 -in guidewire placed through the PJ stricture. The retained guidewire was successfully grasped with the forceps and removed ( Fig.2). The stricture in the pancreaticojejunostomy was managed with balloon dilation and transmural and transanastomotic placement of a 7-Fr double-pigtail plastic stent.

EUS-PG may be useful when transpapillary access is unavailable. However, various complications can occur, such as pancreatitis [1,2]. Guidewire shearing is not uncommon and is regarded as a serious, reportable adverse event $[3,4]$ that can lead to complications. In the current case, EUS-guided pancreatic drainage with a fully covered, self-expandable metal stent allowed opening of the high profile fistula and pancreatic duct for simultaneous removal of the retained guidewire and treatment of the pancreaticojejunostomy stricture. This case may help with the future management of guidewire shearing complications associated with EUS-guided procedures for drainage of the pancreatic duct. 
Endoscopy_UCTN_Code_CPL_1AL_2AD

Competing interests: None

Hoonsub So, Do Hyun Park, Kyoungwon Jung, Sang Soo Lee, Dong Wan Seo, Sung Koo Lee, Myung-Hwan Kim

Division of Gastroenterology, Department of Internal Medicine, University of Ulsan College of Medicine, Asan Medical Center, Seoul, Korea

\section{References}

1 Ergun M, Aouattah T, Gillain C et al. Endoscopic ultrasound-guided transluminal drainage of pancreatic duct obstruction: long-term outcome. Endoscopy 2011; 43: 518-525

2 Shah JN, Marson F, Weilert $F$ et al. Singleoperator, single-session EUS-guided anterograde cholangiopancreatography in failed ERCP or inaccessible papilla. Gastrointest Endosc 2012; 75: 56-64

3 Sharma M, Toshniwal J, Vashistha $C$ et al. Shearing of the sheath of the guide wire: a complication of endoscopic ultrasoundguided rendezvous procedure. Endosc Ultrasound 2013; 2: 171-172

4 National Quality Forum. Serious reportable events in healthcare - 2006 update. Washington, DC: National Quality Forum (NQP); 2007

\section{Bibliography}

DOI http://dx.doi.org/

10.1055/s-0034-1392789

Endoscopy 2015; 47: E458-E459

(c) Georg Thieme Verlag KG

Stuttgart · New York

ISSN 0013-726X

\section{Corresponding author} Do Hyun Park, MD, PhD

Division of Gastroenterology

Department of Internal Medicine Asan Medical Center

88, Olympic-Ro 43-Gil

Songpa-gu

Seoul 138-736

Korea

Fax: +82-2-476-0824

dhpark@amc.seoul.kr 